\title{
Time-of-Day Could Affect Cognitive Screening Performance in Older Patients with TIA and Stroke
}

\author{
Sara Mazzucco Linxin Li Maria A. Tuna Sarah T. Pendlebury Rhoda Frost \\ Rose Wharton Peter M. Rothwell on behalf of the Oxford Vascular Study \\ Centre for Prevention of Stroke and Dementia, Nuffield Department of Clinical Neurosciences, John Radcliffe \\ Hospital, University of Oxford, Oxford, UK
}

\author{
Keywords \\ Cerebrovascular disease · Cognitive impairment · Aging · \\ Circadian rhythm
}

\begin{abstract}
Background and Purpose: The impact of time-of-day on the cognitive performance of older patients with limited cognitive reserve after a transient ischemic attack (TIA) or stroke, and on short cognitive tests, such as the Montreal Cognitive Assessment (MoCA), is unknown. We retrospectively studied whether morning versus afternoon assessment might affect the classification of patients aged 70 or older as severe $(\mathrm{SCl})$, mild $(\mathrm{MCl})$, and no $(\mathrm{NCl})$ cognitive impairment by the MoCA. Methods: Morning (12 p.m. or earlier) versus afternoon (later than 12 p.m.) proportions of $\mathrm{SCl}$ (MoCA score <20), $\mathrm{MCl}$ (MoCA score 25-20) and $\mathrm{NCl}$ (MoCA score $\geq 26$ ) were compared in a cohort of patients aged $\geq 70$, attending a rapidaccess TIA/stroke clinic. Results: Of 278 patients, 113 (40.6\%) were tested in the morning and 165 (59.4\%) in the afternoon. The proportion with $\mathrm{SCl}$ was greater in the afternoon than in the morning ( 10.9 vs. $1.8 \%$, respectively, $p=0.004$ ), with no difference in age, education, diagnosis, disability, or vascular risk factors. Conclusions: Time-of-day appears to affect cognitive performance of older patients after they undergo TIA and minor stroke. If our cross-sectional findings are con-
\end{abstract}

\begin{tabular}{ll}
\hline KARGER & $\begin{array}{l}\text { ( } 2017 \text { The Author(s) } \\
\text { Published by S. Karger AG, Basel }\end{array}$ \\
$\begin{array}{l}\text { E-Mail karger@karger.com } \\
\text { www.karger.com/ced }\end{array}$ & $\begin{array}{l}\text { This article is licensed under the Creative Commons Attribution } 4.0 \\
\text { International License (CC BY) (http://www.karger.com/Services/ } \\
\text { OpenAccessLicense). Usage, derivative works and distribution are } \\
\text { permitted provided that proper credit is given to the author and the } \\
\text { original publisher. }\end{array}$
\end{tabular}

Sara Mazzucco

Centre for Prevention of Stroke and Dementia

Nuffield Department of Clinical Neurosciences, John Radcliffe Hospital

University of Oxford, Level 6, West Wing, Oxford OX3 9DU (UK)

E-Mail sara.mazzucco@ndcn.ox.ac.uk firmed in cross-over studies with repeated testing, timing of assessments should be considered in clinical practice and in research studies.

(c) 2017 The Author(s)

Published by S. Karger AG, Basel

\section{Introduction}

Many trials look at the effect of stroke and stroke-preventive treatments on cognitive trajectory and on the transition from mild cognitive impairment (MCI) to dementia [1]. The reliable distinction between the 2 cognitive states and its reproducibility is therefore important [2]. Differences between diagnostic criteria for MCI can have an impact on the prevalence and rate of progression to dementia [3], but operational differences within wellestablished criteria can also result in significant variation in MCI estimates [2]. For example, it is known that there is diurnal variation in cognitive abilities, and that after 60 , cognitive performance tends to worsen through the day $[4,5]$. In patients with limited cognitive reserve, such as those with MCI, this variation might be clinically relevant. However, according to the extent of our knowledge, no studies on stroke or transient ischemic attack (TIA) have reported on the consistency of timing of cognitive screening, and there are no studies on the impact of time- 
Table 1. Characteristics of 278 patients assessed in the morning versus afternoon

\begin{tabular}{lrrr}
\hline & $\begin{array}{c}\text { Morning } \\
(n=113)\end{array}$ & $\begin{array}{c}\text { Afternoon } \\
(n=165)\end{array}$ & $p$ value \\
\hline Age, years, mean (SD) & $78.12(5.65)$ & $78.19(5.51)$ & 0.925 \\
mRS at 1 month $>1$ & $34(30.1)$ & $46(29.1)$ & 0.483 \\
Low education & $62(54.9)$ & $95(57.6)$ & 0.373 \\
Diagnosis, $n$ (\%) & $76(67.3)$ & $101(61.2)$ & 0.352 \\
$\quad$ TIA & $30(26.5)$ & $46(27.9)$ & \\
Stroke & $7(6.2)$ & $18(10.9)$ & 0.117 \\
Non-vascular & $68(60.2)$ & $112(67.9)$ & 0.439 \\
Risk factors, $n$ (\%) & $18(15.9)$ & $24(14.5)$ & 0.410 \\
$\quad$ History of hypertension & $42(37.5)$ & $57(35.4)$ & 0.090 \\
History of diabetes & $64(57.1)$ & $78(48.1)$ & 0.094 \\
History of hyperlipidaemia & $12(10.6)$ & $28(17)$ & \\
History of smoking & & & \\
Atrial fibrillation & & & \\
\hline
\end{tabular}

Data are number (\%) unless otherwise stated. mRS, modified Rankin Scale; Low education, education $\leq 12$ years.

of-day on cognitive performance as assessed by short cognitive tests, which are increasingly used both in clinical trials and in clinical practice to measure cognitive outcomes [2].

Among short cognitive tests used in patients with cerebrovascular disease, the Montreal Cognitive Assessment (MoCA) test has proved to be one of the most sensitive and specific for MCI, and it incorporates executive and attentional tasks suitable for vascular cognitive impairment $[2,6,7]$. In a cohort of patients aged $\geq 70$ attending a rapid-access TIA/stroke clinic, we did a retrospective cross-sectional study to determine whether there was any evidence to suggest that time-of-day of assessment might affect the categorisation of patients as "severe cognitive impairment" (SCI) as compared to "MCI" or "no cognitive impairment" (NCI) by the MoCA.

\section{Materials and Methods}

\section{Participants}

Consecutive eligible patients aged $\geq 70$, followed-up 1 month after attending the Oxford Vascular Study (OXVASC) rapid-access TIA/stroke clinic between November 2011 and January 2016 were enrolled in this study. The OXVASC Study is an ongoing population-based study on the incidence and outcome of all acute vascular events in a population of 92,728 individuals registered with 100 primary care physicians in 9 practices in Oxfordshire, United Kingdom. Multiple methods of ascertainment are used for patients with TIA or stroke [8], including a daily, rapid-access TIA/stroke clinic, to which participating physicians and the local emergency department refer all individuals with suspected TIA or minor stroke [9].

Cognitive Performance and Time-of-Day in Older Patients after TIA and Stroke

\section{Procedures}

Patients were assessed and treated in the acute phase by a neurologist or stroke physician and all presentations and investigations were reviewed by the senior study neurologist (P.M.R.) as discussed elsewhere [10]; the MoCA was administered at 1 month follow-up to consenting patients. On the MoCA, SCI was defined as a score $<20, \mathrm{MCI}$ as a score between 25 and 20 , while NCI was defined as a score $\geq 26[6,11,12]$.

The OXVASC study was approved by the local ethics committee and consent was obtained from all participants.

\section{Statistical Analysis}

Consecutive patients aged $\geq 70$ who had been able to undergo cognitive assessment after presumed TIA or minor stroke (NIHSS $\leq 3$ ) were included in the analysis if they had no pre-existing clinical diagnosis of dementia and had no disabling neurological deficit due to previous stroke.

Demographic and clinical characteristics and risk factors of patients in the "morning" and in the "afternoon" group were compared using $\chi^{2}$ test or ANOVA as appropriate. Proportions of patients with SCI, MCI and NCI assessed in the morning (12 p.m. or earlier) versus afternoon (later than 12 p.m.) were analysed using $\chi^{2}$ test.

\section{Results}

Of 279 patients aged $\geq 70$ undergoing cognitive screening with the MoCA 1 month after attending a rapid-access TIA/stroke clinic, one was excluded from the analysis because the time of the cognitive testing was not recorded, leaving 278 patients in the final analysis. Of these, 113 (40.6\%) were tested in the morning and 165 (59.4\%) in the afternoon. The median time of assessment in the morning was 10.55 a.m. (interquartile range [IQR] 10.15 
Table 2. Cognitive performance according to the time-of-day of assessment in 278 patients aged $\geq 70$

\begin{tabular}{lccl}
\hline Study population & Morning, $n(\%)$ & Afternoon, $n(\%)$ & $p$ value \\
\hline NCI (MoCA $\geq 26)$ & $66(58.4)$ & $97(58.8)$ & 0.95 \\
MCI (MoCA 25-20) & $45(39.8)$ & $50(30.3)$ & 0.1 \\
SCI (MoCA <20) & $2(1.8)$ & $18(10.9)$ & 0.004 \\
Total & $113(100)$ & $165(100)$ & 0.008 \\
\hline
\end{tabular}

NCI, no cognitive impairment; MCI, mild cognitive impairment; SCI, severe cognitive impairment.

a.m. -11.30 a.m.), while in the afternoon it was 1.49 p.m. (IQR 12.44 p.m.-2.53 p.m.).

The "morning" and the "afternoon" groups did not differ significantly in terms of age, diagnosis, education, modified Rankin Scale, and risk factors (Table 1).

Overall, 163 patients were classified as NCI, 95 as MCI and 20 as SCI. While there was no significant difference in morning vs. afternoon proportion of NCI $(p=0.95)$ or MCI $(p=0.1)$, patients assessed in the afternoon were significantly more likely to display SCI than patients who were assessed in the morning ( $p=$ 0.004; Table 2).

\section{Discussion}

To our knowledge, this is the first study to explore the effect of time-of-day on cognitive performance as assessed by short cognitive tests, such as the MoCA, and the first study in older patients with TIA and stroke. Short tests are increasingly used not only in clinical practice to follow-up patients with cognitive impairment but also in large multicentre clinical trials both as screening tools and as cognitive outcomes [1]. To our knowledge, previous clinical trial protocols have not required consistent timing of cognitive assessments.

In our study, patients tested in the afternoon were more likely to be classified as SCI as compared to patients tested in the morning. This could be consistent with our hypothesis that older patients with limited cognitive reserve might be more susceptible to cognitive fluctuations triggered by environmental factors, including time-ofday. Previous studies have suggested that in older subjects, cognitive performance tends to worsen through the day, while in younger people it is worse early in the morning and improves along the day [5].

Our study has limitations. First, our analysis was purely cross-sectional and our findings need to be confirmed through cross-over studies with repeated assessments at different times of day. Our patients had had a prior cognitive assessment in the acute phase after their TIA or stroke, but this had usually been in the morning only and was also confounded by acute-phase effects $[10,13]$, undermining the interpretation of any differences from the 1-month follow-up assessments reported here. However, our findings have highlighted the potentially important but neglected the issue of the timing of assessment, and the need for further prospective studies required to formally test them. Second, we were able to study diurnal variation in cognition only during office hours ( 9 a.m.5 p.m.). Although this period is probably most relevant in relation to clinical assessments and research studies, reduced cognitive performance in the evening could be a greater clinical problem.

In conclusion, time-of-day appears to affect cognitive performance of older patients after TIA and minor stroke. If our findings are confirmed in cross-over studies with repeated assessments, timing of testing would be particularly important in implementing research protocols in studies with repeated cognitive assessments after TIA or stroke; these are studies that predominantly involve older patients.

\section{Acknowledgements}

We acknowledge the use of the facilities of the Cardiovascular Clinical Research Facility, Oxford.

\section{Sources of Funding for the Study}

The Oxford Vascular Study has been funded by the Wellcome Trust, Wolfson Foundation, UK Stroke Association, British Heart Foundation, National Institute of Health Research (NIHR), Dunhill Medical Trust and the NIHR Biomedical Research Centre, Oxford. Dr. L. Li was funded by the China Scholarship Council. 
Dr. M.A. Tuna was funded by the Gulbenkian Foundation Doctoral Programme for Physicians. Prof. S.T. Pendlebury was funded by the Oxford NIHR Biomedical Research Centre. Prof. P.M. Rothwell is in receipt of an NIHR Senior Investigator Award and a Wellcome Trust Senior Investigator Award.

\section{Disclosure Statement}

The authors have no disclosures to make.

\section{References}

$>1$ Tzourio C, Anderson C, Chapman N, Woodward M, Neal B, MacMahon S, Chalmers J; PROGRESS Collaborative Group: Effects of blood pressure lowering with perindopril and indapamide therapy on dementia and cognitive decline in patients with cerebrovascular disease. Arch Intern Med 2003;163:1069-1075.

-2 Pendlebury ST, Mariz J, Bull L, Mehta Z, Rothwell PM: Impact of different operational definitions on mild cognitive impairment rate and MMSE and MoCA performance in transient ischaemic attack and stroke. Cerebrovasc $\mathrm{Dis} 2013 ; 36: 355-362$.

-3 Busse A, Bischkopf J, Riedel-Heller SG, Angermeyer MC: Subclassifications for mild cognitive impairment: prevalence and predictive validity. Psychol Med 2003;33:10291038.

4 Blatter K, Cajochen C: Circadian rhythms in cognitive performance: methodological constraints, protocols, theoretical underpinnings. Physiol Behav 2007;90:196-208.

5 Yoon C, May CP, Hasher L: Aging, circadian arousal patterns, and cognition; in Schwarz NPD, Knauper B, Sudman S (eds): Aging, Cognition and Self-Reports. Washington, Psychological Press, 1999.
6 6 Pendlebury ST, Cuthbertson FC, Welch SJ, Mehta Z, Rothwell PM: Underestimation of cognitive impairment by Mini-Mental State Examination versus the Montreal Cognitive Assessment in patients with transient ischemic attack and stroke: a population-based study. Stroke 2010;41:1290-1293.

7 Nasreddine ZS, Phillips NA, Bedirian V, Charbonneau S, Whitehead V, Collin I, Cummings JL, Chertkow H: The Montreal Cognitive Assessment, MoCA: a brief screening tool for mild cognitive impairment. J Am Geriatr Soc 2005;53:695-699.

8 Rothwell PM, Coull AJ, Giles MF, Howard SC, Silver LE, Bull LM, Gutnikov SA, Edwards P, Mant D, Sackley CM, Farmer A, Sandercock PA, Dennis MS, Warlow CP, Bamford JM, Anslow P; Oxford Vascular Study: Change in stroke incidence, mortality, casefatality, severity, and risk factors in Oxfordshire, UK from 1981 to 2004 (Oxford Vascular Study). Lancet 2004;363:1925-1933.

\9 Li L, Yiin GS, Geraghty OC, Schulz UG, Kuker W, Mehta Z, Rothwell PM; Oxford Vascular Study: Incidence, outcome, risk factors, and long-term prognosis of cryptogenic transient ischaemic attack and ischaemic stroke: a population-based study. Lancet Neurol 2015; 14:903-913.

10 Mazzucco S, Li L, Tuna MA, Pendlebury ST, Wharton R, Rothwell PM; Oxford Vascular Study: Hemodynamic correlates of transient cognitive impairment after transient ischemic attack and minor stroke: a transcranial Doppler study. Int J Stroke 2016;11:978986.

11 Webb AJ, Pendlebury ST, Li L, Simoni M, Lovett N, Mehta Z, Rothwell PM: Validation of the Montreal Cognitive Assessment versus Mini-Mental State Examination against hypertension and hypertensive arteriopathy after transient ischemic attack or minor stroke. Stroke 2014;45:3337-3342.

12 Trzepacz PT, Hochstetler H, Wang S, Walker B, Saykin AJ; Alzheimer's Disease Neuroimaging Initiative: Relationship between the Montreal Cognitive Assessment and Mini-Mental State Examination for assessment of mild cognitive impairment in older adults. BMC Geriatr 2015;15:107.

13 Pendlebury ST, Wadling S, Silver LE, Mehta Z, Rothwell PM: Transient cognitive impairment in TIA and minor stroke. Stroke 2011; 42:3116-3121. 